\title{
A Novel Approach for Image Recognition to Enhance the Quality of Decision Making by Applying Degree of Correlation Using Artificial Neural Networks
}

\author{
Raju Dara \\ Research Scholar, Department of Computer Science and Engineering \\ Jawaharlal Nehru Technological University Kakinada, Andhra Pradesh, India \\ rajurdara@gmail.com \\ Dr. Ch.Satyanarayana \\ Research Scholar, Department of Computer Science and Engineering \\ Jawaharlal Nehru Technological University Kakinada, Andhra Pradesh, India \\ chsatyanarayana@yahoo.com \\ Dr. A. Govardhan \\ Professor, Department of Computer Science and Engineering \\ Jawaharlal Nehru Technological University, Hyderabad. Andhra Pradesh, India \\ govardhan_cse@yahoo.co.in
}

\begin{abstract}
Many diversified applications do exist in science \& technology, which make use of the primary theory of a recognition phenomenon as one of its solutions. Recognition scenario is incorporated with a set of decisions and the action according to the decision purely relies on the quality of extracted information on utmost applications. Thus, the quality decision making absolutely reckons on processing momentum and precision which are entirely coupled with recognition methodology. In this article, a latest rule is formulated based on the degree of correlation to characterize the generalized recognition constraint and the application is explored with respect to image based information extraction. Machine learning based perception called feed forward architecture of Artificial Neural Network has been applied to attain the expected eminence of elucidation. The proposed method furnishes extraordinary advantages such as less memory requirements, extremely high level security for storing data, exceptional speed and gentle implementation approach.
\end{abstract}

Index Terms - Image recognition, information retrieval, artificial neural network, degree of correlation, faulttolerance.

\section{INTRODUCTION}

With the advancement of digital technology in the present life without involvement of the image is virtually not possible. This is often as a result of, image carries clear data in most of the cases not solely that our brain has a lot of stable structure to investigate and perceive the image from a psychological point of observation. For operational perspective, recognition of an image is extremely difficult and computationally expansive downside. The challenges offered by recognition method are accuracy, operational speed, storage demand and quality in implementation. With recognition in different fields, varied applications are integrated like in healthcare, automotive, business, internet based applications and advertisements, etc. In this paper a technique is proposed to recognize the image from a database wherever it is stored and in result varied activities will outline according to requirements like just in case of image based data retrieval or in industrial automation. Proposed methodology has extension of capability of recognition for image with its outlier variations and with its partial data. Developed approach has given attention to deliver the great performance even with faults are offered with solution. This sort of quality is enormously crucial for hardware characterization wherever faults are inherent characteristics with the moment.

\section{A. Back ground}

Altogether the image recognition techniques planned and require pre-processing of image, feature extraction stage and artificial neural network for classification purpose [1]. Pre-processing is an important step that enhances the quality and produces an image in which minutiae can be detected correctly [2]. A data cleaning graph with data quality constraints is used to help users in identifying the points of the graph, and the records need the attention towards manual data repairs to represent the 
required feedback to clean data items manually [3]. In this paper, three major data mining methods, namely functional dependency mining, association rule mining and Bagging SVMs for data cleaning are discussed [4]. In this article, the system uses the extract, transform and load methods as the main process model, apart from these, the techniques applied to scrub the data are: parsing technique to identify the dirty data, the regular expression method for matching attributes, and k-Nearest Neighbor algorithm [5]. Organizational databases are comprehended with data of poor quality, this paper develops a framework that consists of seven elements: management responsibilities, operation and assurance costs, research and development, production, distribution, personnel management, and legal function to analyze data quality [6]. Information quality (IQ) has become a critical concern of organizations and an active area of Management Information Systems (MIS) research, the methodology which is used here called as AIMQ (AIM Quality) to form a basis for IQ assessment and benchmarking [7]. This article presents a methodology to test its efficacy through a rigorous case study and contributes two key features such as, first one is developing a 2 X 2 conceptual model for describing IQ which refers to this model as the product \& service performance model for information quality (PSP/IQ), and next is integrating the IQ dimension. PSP/IQ model provides a way to compare information quality across organizations, and to develop IQ benchmarks [8]. An innovative technique is supposed to be implemented and adopted to deal with data quality issues where organizational databases are enormous, in this case the possible solution to maintain hygienic and stagnant information in the database is the conversion of preprocessed and scrubbed data into image. Henceforth do perform image recognition techniques to extract the required data. The definition for image recognition can be given as the identification of objects in an image and this process would probably start with image processing techniques such as noise removal followed by (low-level) feature extraction to locate lines [9], regions and possibly areas with certain textures. This paper presents a real time or online method for recognition and verification handwritten signatures by using NN architecture [10]. In this work, the authors used back propagation neural network for the implementation of image recognition scenario. The proposed system performs human face recognition at a very high degree of accuracy [11]. The main focus is on frontal two dimensional images that are taken in a controlled environment i.e. the illumination and the background will be constant. All the other methods of person's identification and verification like iris scan or finger print scan require high quality and costly equipment's but in face recognition we only require a normal camera giving us a 2-D frontal image of the person that will be used for the process of the person's recognition [12]. Most connectionist approaches rely on the use of simple MLPs and the relationships between different uses of ANNs in different tasks [13]. In this paper, the authors used
Multilayer perceptrons that are trained in a supervised way using a set of simulated noisy images together with the corresponding clean images for the desired output [14]. In this approach, Multilayer perceptrons (MLP) are trained in supervised way of learning, methods using a cleaning image product with threshold technical and the corresponding noise handwritten image that product from scanning with scan machine [15].A feature extractor is designed based on Mutual Information computation. The Neural Networks is used with two hidden layers and one output layer with 3 nodes that correspond to varieties (classes) of FIG leaves. The proposal technique is a tester on a database of 84 images leaves with 28 images for each variety (class) [16]. In this work, the authors used Image Receptive Fields Neural Network (IRF-NN) for image recognition [17]. By the character of the Hopfield neural network, associative memory supported this mechanism will be effectively adapted for the image recognition application. It is proposed that the design and prototype implementation of image recognition through the discrete Hopfield neural network [18]. The proposed approach will recover the dirty image like fragment or an uproarious image with smart potency. A technique for applying Hopfield neural network (HNN) with grey pictures has bestowed in [19]. The quality of automatic recognition of pulse pictures within the analysis of processed pulse identification is bestowed [20]. A fuzzy neural network for classifying pulse images based on the data of consultants in ancient Chinese pulse diagnosis was designed, so as to automatically differentiate the pulsate patterns using tiny samples in pulse identification. Within the region of agriculture, [21] has given utilization in recognition for the classification of traditional and unsound wheat. The main objective of this research is to develop rule to acknowledge unsound wheat based image processing and artificial neural network. To mark the catalyst activity supported BP neural network is proposed here in [22] to complement the recognition strategies. Complications of blurred image recognition, employ a multilayer neural network based on multi-valued neurons (MLMVN) and has been measured in [23]. Use of the frequency domain as a feature space has taken, since the Fourier section spectrum of a blurred image remains virtually unaffected, a minimum of within the low frequency component, it is feasible to use phases corresponding to the lowest frequencies as an option for recognition. The proposed technique in [24] states that the dynamics of neurons are decided as well as geometrical native options obtained from the Dennis Gabor filtering then the image regions beneath resultant neural synchronization are identified. Software and hardware approach using features extracted from the wavelet transform (WT) of the image as input to a pattern classifier has bestowed on [25]. The wavelet transform will be calculated via adaptive neural network, whereas the pattern classification could be administrated by an adaptive fuzzy neural network, hence the systems are going to be absolutely parallel and distributed. Paper [26] explores the recognition of number, image of fastening pixels based on the neural network ensemble. Initially, 
the bagging technique is employed to get the training samples, subsequently, the discrete Hopfield neural network is exploited to get rid of the noise among the samples and associated samples, then twenty single 3 layer feed forward neural networks are designed to construct a neural network ensemble to get the recognition results through a vote. It is clearly mentioned that an information retrieval with an image from database using an image as an input taken from my phone mentioned in [27].

This paper is organized as follows: in section II, explained the proposed principle of recognition, in section III, discussed proposed recognition techniques. Section IV describes fault tolerance analysis of artificial neural network system, Section V provides experimental results and simulation. Section VI states the advantages of proposed system and finally concluded in section VII.

\section{PROPOSED PRINCIPLE OF RECOGNITION}

\section{A. Basic principle: Degree of Correlation (DoC)}

Suppose the interaction of two parameters takes place under diversified environments and circumstances in such cases too, the resultant outcomes must be a unique value, then this distinctive class of unique value can be defined as the degree of correlation (DoC) between the parameters. Mathematically, this can be formulated as follows:

$$
\varphi\left(\mathrm{U}_{\mathrm{i}}, \mathrm{V}\right) \neq \varphi\left(\mathrm{U}_{\mathrm{j}}, \mathrm{V}\right) ; \forall \mathrm{j} \text { if } \mathrm{i} \neq \mathrm{j}
$$

Where Ui is external stimulus and has an established correlation with environment $\varphi$ that contains parameter $\mathrm{V}$, and $\mathrm{Uj}$ is a new test input in the same environment. This principle is also valid, if stimulus is same for different parameter available in the environment and this can be expressed as follows:

$$
\varphi\left(\mathrm{U}, \mathrm{V}_{\mathrm{i}}\right) \neq \varphi\left(\mathrm{U}, \mathrm{V}_{\mathrm{j}}\right) ; \forall \text { j if } \mathrm{i} \neq \mathrm{j}
$$

This degree of correlation can be utilized for recognition point of view in varied applications of pattern recognition, particularly within the field of image recognition where recognition process has been cascaded with automatic action as response of recognition.

\section{B. Neural Modeling of Correlation}

The above defined notion of degree of correlation can efficiently be modelled with the artificial neural networks. Let the environment obtained by the feed forward neural network architecture consists of multilayer structure with single output node and unimodel signed as activation function. Required circumstance of the maximum degree of correlation between stimulus $U$ and available connection weights may be established by applying the learning. After having the training of weights in neural parameter, $\mathrm{V}$ appeared as trained weight $\mathrm{W}$. The neural modeling of (2) for recognition of input image $U_{i}$ ' has a lot of statistical and physical similarity characteristics with image $U_{i}$ compare to image $U_{j}$ and may be outlined as

$$
\begin{aligned}
& \left|f\left(\Phi\left(\mathrm{U}_{\mathrm{i}}, \mathrm{W}_{\mathrm{i}}\right)\right)-f\left(\Phi\left(\mathrm{U}_{\mathrm{i}}^{\prime}, \mathrm{W}_{\mathrm{i}}\right)\right)\right|< \\
& \left|f\left(\Phi\left(\mathrm{U}_{\mathrm{j}}, \mathrm{W}_{\mathrm{j}}\right)\right)-f\left(\Phi\left(\mathrm{U}_{\mathrm{i}}, \mathrm{W}_{\mathrm{j}}\right)\right)\right| ; \forall \mathrm{i}, \mathrm{j} \text { if } \mathrm{i} \neq \mathrm{j}
\end{aligned}
$$

Where $\mathrm{W}_{\mathrm{i}}$ and $\mathrm{W}_{\mathrm{j}}$ are set of trained weights corresponding to image $U_{i}$ and $U_{j}$.In fact the philosophy of learning can be defined as establishment of relationship w.r.t object and this can be attainable by iterative adjustment of neural weights in association of input information for fixed value of target.

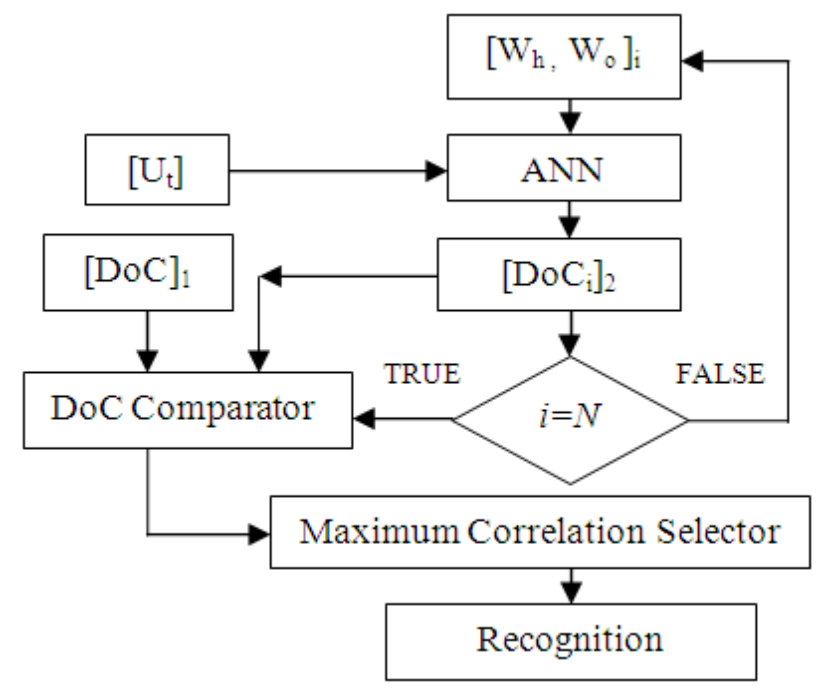

Fig 1. Forced recognition

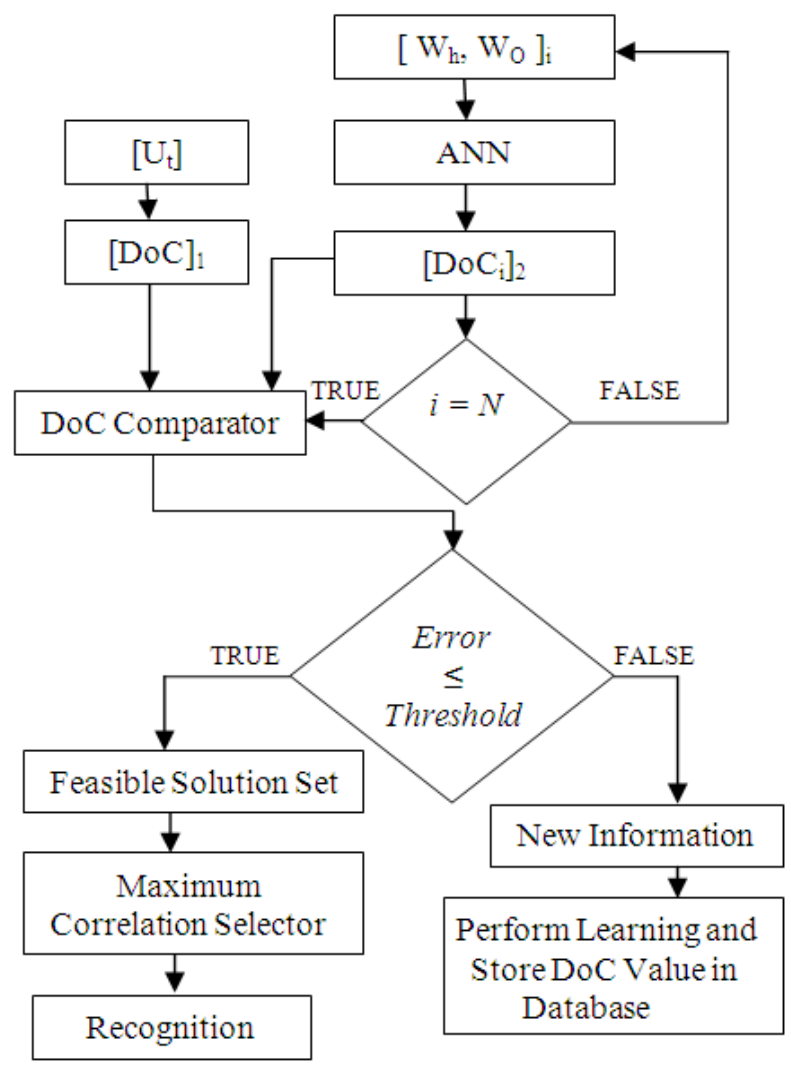

Fig 2. Custom recognition 


\section{Proposed ReCognition TECHNIQUE}

Recognition may be a recalling method during which past expertisation employs to classify and acknowledge the object. If the object does exist inside the domain of occurrence, then the recalling process is error free, otherwise an error value. Acceptable values of error reckons upon application. In this paper, we divided the task of recognition into two categories, namely "Forced recognition and Custom recognition".

\section{A. Forced Recognition}

This class of recognition is applied when a recognition decision has to be given regardless of applied input and error in the result i.e. forcibly recognition obtained from solution. Conception of decision is taken in terms of max degree of correlation among potentialities accessible with learning information. This type of method is appropriate where there is no time available to acquire the new knowledge and decision has to be taken only with available knowledge. In forced recognition wherever the decision is taken in favor of things that have been learned antecedently using the max correlation method as shown in Fig -1 .

\section{B. Custom Recognition}

Here decision doesn't belong to the domain of expertization, if the object has not been learned earlier, therefore recalling may generate an error. A predefined threshold of error is used to determine the acceptability of error in recalling. If it is less than the threshold, max correlation is employed to take the decision otherwise the object will be declared as new information. This category is appropriate, wherever preciseness in result is exceptionally significant or time is available to get the learning, if the unknown information available at present or both. Working process has been shown in fig-2. In both the cases of recognition, $\mathrm{DoC}_{1}$ represents the obtained degree of correlation after learning.

\section{FAUlt TOLERANCE ANALYSIS OF ARTIFICIAL NEURAL NETWORK SYSTEM}

When a system design is accomplished, then the quality characteristics can be outlined in in varied ways like speed, power consumption, size, etc. Another vital parameter is fault tolerance, which exactly defines the reliable and the required output of the system even in fault conditions too. It is required to analyze the system thoroughly to know the performance, significance with respect to fault tolerance, for a given design. Artificial Neural Networks work on parallel distributed computing, so that the expectation of fault tolerance may be incredibly high. This can be the issue to know how faults in ANN show its impact on performance with the conferred principle of recognition. From a functional point of view and independently of the hardware implementation options, an Artificial Neural Networks may lead to the subsequent faults: i. Fault within a connection or weight or multiplier

ii. Fault in an input

iii. Fault in a multiplier, adder or accumulator

iv. Fault within the activation function

Some of these faults will mask one another within the sense that it may be indistinguishable what kind of a fault is occurring. This would the case of faults of type (iii) and (iv) that produces the produces a constant kind of impact within the output and might be considered as the identical state of issues. While other faults occur in elements of the network that have connections in parallel and the impact in the output must be analyzed, in this part of the circuit the connection is serial and the effect is similar. Faults of type (i) and (iii), both include the multiplier and this duplication is measured as a result of the existence of a multiplier associated with each connection falls into fault type (i) and the existence of a single multiplier before the activation function falls in to fault type (iii). Among all attainable faults, faults with weights and connections are a lot of sense from a practical point of view, hence the analysis has been performed after that. The global model to be used and consists of faults of type stuck at $+\mathrm{W}_{\max },-\mathrm{W}_{\max }$ or zero for all the connections, wherever $+\mathrm{Wmax}$ is the $\max$ value that the weight can be assumed with practical effect. This model must be employed in the subsequent method: Test the effect of each individual fault by setting the weight associated with each connection to $+\mathrm{W}_{\max }$, $\mathrm{W}_{\max }$ and zero. Practically faults prevalence happens with uniform distribution over the connection, but with the proposed design there may be a probability to have faults in hidden layer where connections are incredibly huge (0.9811) comparable to output connections, thus the solution ought to have the robustness against faults in hidden layer connections.

\section{Simulation}

The proposed formulated principle has been applied in this paper for fixed image recognition with and without a variety of noises. In fact, it has more inclination towards application in data mining such data cleaning where the information has to be retrieved with fixed image as an input. To prove the principle by experimentation ten totally different grayscale images are taken into consideration, each encompasses a size of $512 * 512$ pixels. Preprocessing is applied to each image in terms of normalization, noise removal etc., and dividing each image as a set of $8 * 8$ pixel block. A feed forward neural architecture consists of sixty four input nodes, four hidden nodes and one output is created. Bias with fixed input +1 , has also applied to hidden nodes and output nodes. All weights are initialized as a random number by a uniform distribution in the range of [-1 1]. A separate learning (a fixed number of iterations equal to 5) methodology is employed using back-propagation algorithm for each individual image with the target equal to 1 . Momentum is additionally applied to momentum constant equal to 0.1 to increase the learning speed and 
the value of learning rate is taken as 0.1 for all the images. Once the learning of an image is completed, then the DoC for each block can be taken as the output, which is produced by the neural network for that block and stored in an array that is outlined for individual image. In the result, an array corresponding to each image carries an abundant variety of DoC values as several blocks available in an image and the trained weights corresponding to each image are also stored. In other words, for each image there exists a corresponding array which consists of DoC and a set of trained weights in memory.

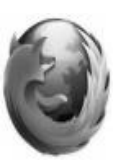

(i)

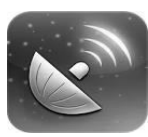

(vi)

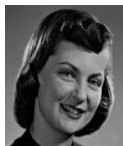

(ii)

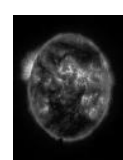

(iii)

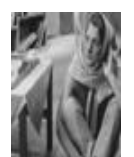

(iv)

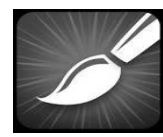

(vii)

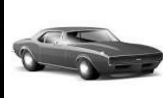

(viii)

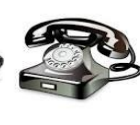

(ix)

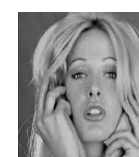

(v)

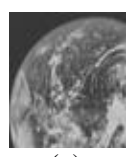

(x)
Fig 3. Images in Database

In the test case, preprocessing techniques such as normalization and noise removal can be applied whenever any one of the trained images with or without variations has been considered as an input to system for recognition and the image can be partitioned into same size of blocks (i.e., $8 * 8$ ). DoC is calculated with each set of stored weights and the absolute distinction in correlation is obtained with each stored correlation value. Position of min total difference established was considered as the final recognition of an image and corresponding action outlined consequently reckons on the nature of recognition either forced or custom. Within the forced recognition, the position with min distinction is the answer whereas the custom recognition depends on defined threshold value. Images taken for simulation to create the data set and their DoC values are stored in the same order as stated in Fig-3.

Color histogram method is applied for comparison purpose which is frequently used for content based image retrieval applications. Reasons for extensive utilization of this approach are trivial to compute, simplicity in its presentation, and exhibit enticing properties. Color histograms do not have inclination towards spatial information with the pixels of a given color because spatial information is largely invariant to the rotation and translation of objects in the image. Color histograms are robust against blockage and changes in camera viewpoint.

Performance for distinct cases have been mentioned: Two additional parameters are used to articulate the excellence of results along with recognition performance as relative distance from mean index (RDMI) that is percentage error in recognition from mean error value with all images in the database and nearest neighbor distance index (NNDI) which is percentage difference of recognize image from nearest neighbor with respect to mean. Positive higher value for each index parameters is fine. Every image of the database has taken and recognition performance shown by the proposed method (DoC). The histogram technique has given in table-1 to table-4. Min error positions in result present the image available within the database as a recognized image for given test image. Test case images are defined under subsequent categories:

\section{A. Test Images are Original Images from the Database}

Table 1. Performance With Original Image

\begin{tabular}{|c|c|c|c|c|c|c|}
\hline \multirow{2}{*}{$\begin{array}{l}\text { Imag } \\
\text { e No. }\end{array}$} & \multicolumn{3}{|c|}{ Degree of Correlation (\%) } & \multicolumn{3}{|c|}{ Histogram method (\%) } \\
\hline & Rec. & RDMI & NNDI & Rec. & RDMI & NNDI \\
\hline 1 & $\sqrt{ }$ & 100 & 55 & $\sqrt{ }$ & 100 & 87 \\
\hline 2 & $\sqrt{ }$ & 100 & 72 & $\sqrt{ }$ & 100 & 50 \\
\hline 3 & $\sqrt{ }$ & 100 & 76 & $\sqrt{ }$ & 100 & 49 \\
\hline 4 & $\sqrt{ }$ & 100 & 80 & $\sqrt{ }$ & 100 & 42 \\
\hline 5 & $\sqrt{ }$ & 100 & 76 & $\sqrt{ }$ & 100 & 81 \\
\hline 6 & $\sqrt{ }$ & 100 & 74 & $\sqrt{ }$ & 100 & 42 \\
\hline 7 & $\sqrt{ }$ & 100 & 76 & $\sqrt{ }$ & 100 & 64 \\
\hline 8 & $\sqrt{ }$ & 100 & 74 & $\sqrt{ }$ & 100 & 91 \\
\hline 9 & $\sqrt{ }$ & 100 & 69 & $\sqrt{ }$ & 100 & 65 \\
\hline 10 & $\sqrt{ }$ & 100 & 84 & $\sqrt{ }$ & 100 & 86 \\
\hline
\end{tabular}

The result of one sample case is shown below
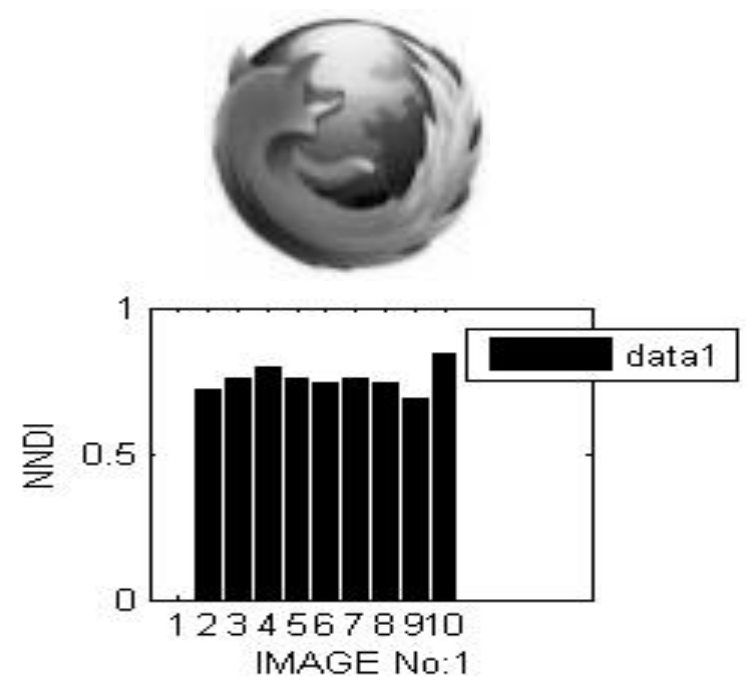

Fig 4. Recognition by DoC for image-i 


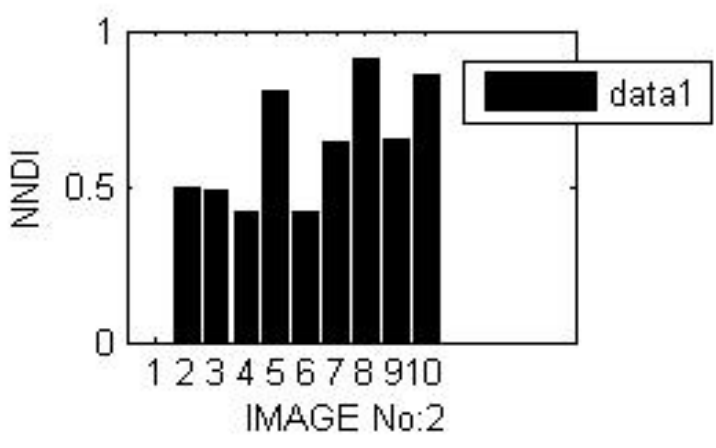

Fig-5. Recognition by Histogram method for image-ii

Performance of proposed DoC and Histogram method for original image in terms of recognition efficiency is well and is shown in table-1.

B. Original Image from Database with Gaussian Distributed Noise (Generated with 0 Mean and 0.01 Variance)

This case is incredibly important for the application purpose, as a result, once images pass through channel then Gaussian noise can be applied. DoC has given excellent performance in contrast with performance given away by the Histogram method that is extremely poor as shown in table -2. This happens because of the Gaussian noise where it completely manipulates the characteristics of the histogram of corresponding images.

Table 2. Performance With Original Image \& Gaussian Noise

\begin{tabular}{|l|l|l|c|l|l|c|}
\hline \multirow{2}{*}{$\begin{array}{l}\text { Image } \\
\text { No. }\end{array}$} & \multicolumn{3}{|c|}{ Degree of Correlation (\%) } & \multicolumn{3}{|c|}{ Histogram method (\%) } \\
\cline { 2 - 7 } & Rec. & RDMI & NNDI & Rec. & RDMI & NNDI \\
\hline 1 & $\sqrt{ }$ & 100 & 38 & $\sqrt{ }$ & 100 & 32 \\
\hline 2 & $\sqrt{ }$ & 100 & 49 & $\sqrt{ }$ & 100 & -25 \\
\hline 3 & $\sqrt{ }$ & 100 & 58 & $\sqrt{ }$ & 100 & 29 \\
\hline 4 & $\sqrt{ }$ & 100 & 10 & $\sqrt{ }$ & 100 & 26 \\
\hline 5 & $\sqrt{ }$ & 100 & 49 & $\sqrt{ }$ & 100 & -28 \\
\hline 6 & $\sqrt{ }$ & 100 & 48 & $\sqrt{ }$ & 100 & -38 \\
\hline 7 & $\sqrt{ }$ & 100 & 57 & $\sqrt{ }$ & 100 & -21 \\
\hline 8 & $\sqrt{ }$ & 100 & 49 & $\sqrt{ }$ & 100 & 35 \\
\hline 9 & $\sqrt{ }$ & 100 & 48 & $\sqrt{ }$ & 100 & -19 \\
\hline 10 & $\sqrt{ }$ & 100 & 68 & $\sqrt{ }$ & 100 & -06 \\
\hline
\end{tabular}

The result of another sample case is shown below

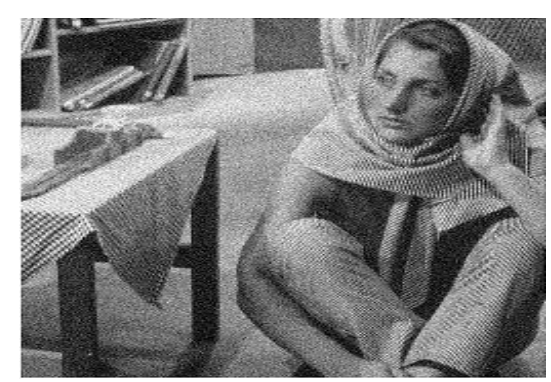

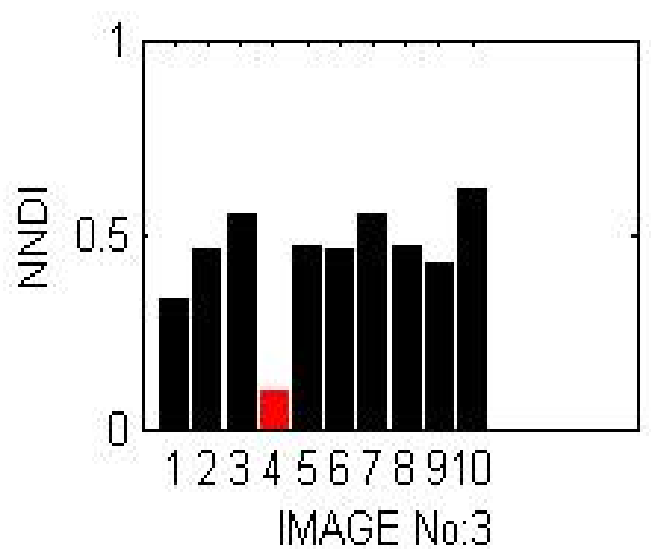

Fig- 6. Recognition by DoC for image-iv

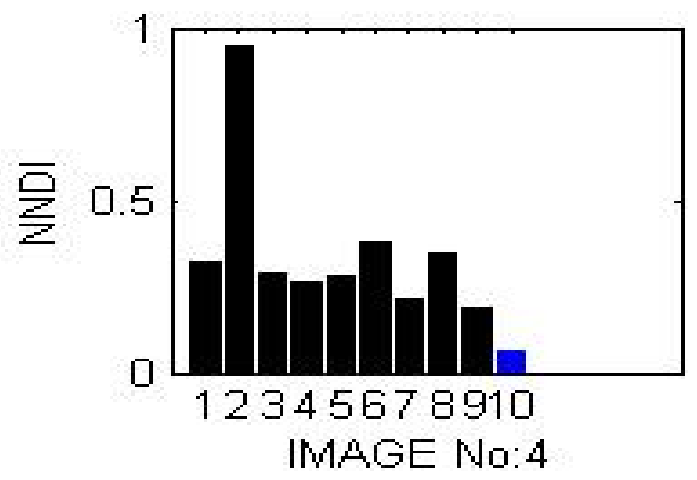

Fig- 7.Recognition by Histogram method for image-iv

C. Original Image from Database with Salt-Pepper Noise (with noise density 5\%)

Table 3. Original Image\& Salt-Pepper Noise

\begin{tabular}{|c|c|c|c|c|c|c|}
\hline \multirow{2}{*}{$\begin{array}{l}\text { Image } \\
\text { No. }\end{array}$} & \multicolumn{2}{|c|}{ Degree of Correlation (\%) } & \multicolumn{3}{|c|}{ Histogram method (\%) } \\
\cline { 2 - 8 } & Rec. & RDMI & NNDI & Rec. & RDMI & NNDI \\
\hline 1 & $\sqrt{ }$ & 75 & 69 & $\sqrt{ }$ & 92 & 79 \\
\hline 2 & $\sqrt{ }$ & 74 & 98 & $\sqrt{ }$ & 84 & 97 \\
\hline 3 & $\sqrt{ }$ & 74 & 51 & $\sqrt{ }$ & 84 & 55 \\
\hline 4 & $\sqrt{ }$ & 66 & 49 & $\sqrt{ }$ & 83 & 49 \\
\hline 5 & $\sqrt{ }$ & 68 & 48 & $\sqrt{ }$ & 87 & 51 \\
\hline 6 & $\sqrt{ }$ & 71 & 47 & $\sqrt{ }$ & 83 & 49 \\
\hline 7 & $\sqrt{ }$ & 76 & 43 & $\sqrt{ }$ & 91 & 49 \\
\hline 8 & $\sqrt{ }$ & 70 & 49 & $\sqrt{ }$ & 87 & 78 \\
\hline 9 & $\sqrt{ }$ & 70 & 42 & $\sqrt{ }$ & 87 & 54 \\
\hline 10 & $\sqrt{ }$ & 72 & 16 & $\sqrt{ }$ & 89 & 18 \\
\hline
\end{tabular}

Both the techniques have shown the confrontation over this sort of noise, but Histogram method is slightly better and shown in table-3.

The result of one sam0ple case is shown below 


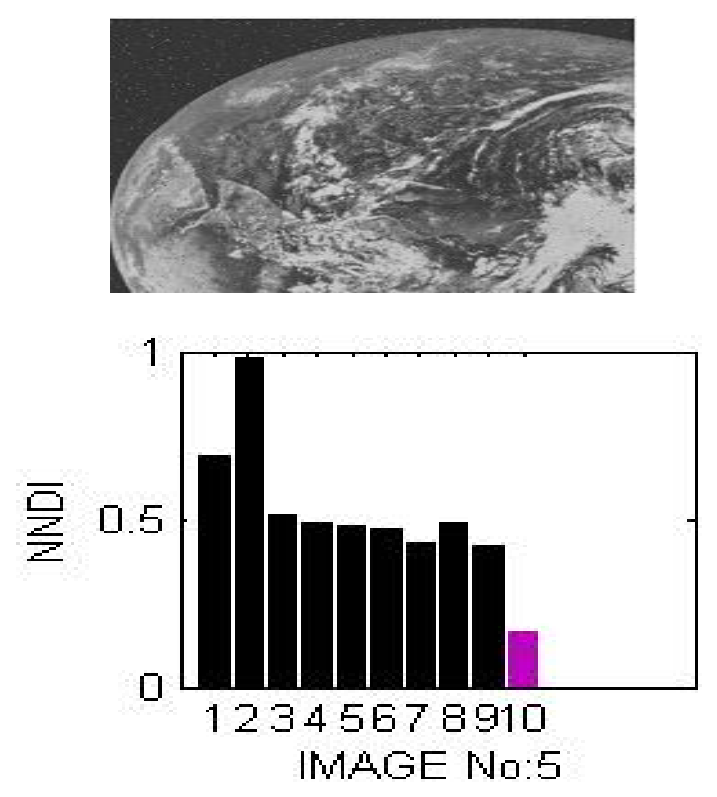

Fig 8. Recognition by DoC for image-x

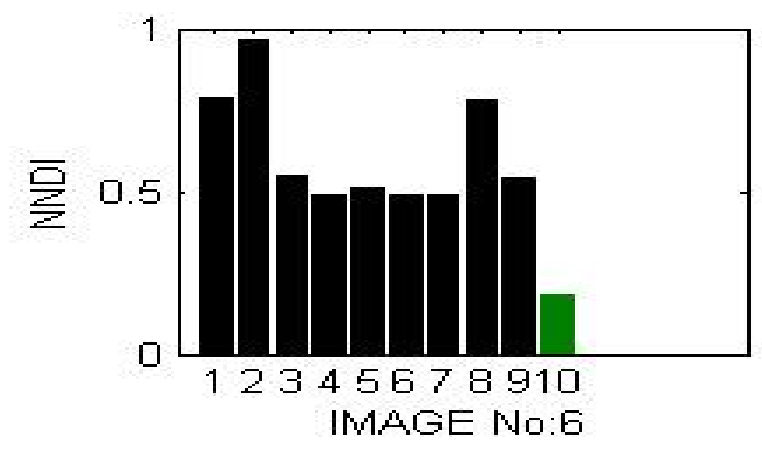

Fig 9.Recognition by Histogram method for image-x

\section{Original Shaded Image from Database}

Table 4. Performance With Shaded Original Image

\begin{tabular}{|c|c|c|c|c|c|c|}
\hline \multirow[t]{2}{*}{$\begin{array}{l}\text { Image } \\
\text { No. }\end{array}$} & \multicolumn{3}{|c|}{$\begin{array}{l}\text { Degree of Correlation } \\
(\%)\end{array}$} & \multicolumn{3}{|c|}{ Histogram method (\%) } \\
\hline & Rec. & RDMI & $\begin{array}{l}\text { NND } \\
\text { I }\end{array}$ & Rec. & RDMI & $\begin{array}{l}\text { NND } \\
\text { I }\end{array}$ \\
\hline 1 & $\begin{array}{l}\lambda[10 \\
]\end{array}$ & -14 & -45 & $\sqrt{ }$ & 16 & 14 \\
\hline 2 & $\sqrt{ }$ & 46 & 16 & $\times[3]$ & 08 & -0.75 \\
\hline 3 & $\sqrt{ }$ & 62 & 37 & $\sqrt{ }$ & 10 & 05 \\
\hline 4 & $\sqrt{ }$ & 42 & 13 & $\sqrt{ }$ & 09 & 0.9 \\
\hline 5 & $\sqrt{ }$ & 72 & 49 & $\sqrt{ }$ & 14 & 12 \\
\hline 6 & $\sqrt{ }$ & 54 & 06 & $\times[8]$ & 09 & -03 \\
\hline 7 & $\sqrt{ }$ & 72 & 76 & $\sqrt{ }$ & 31 & 09 \\
\hline 8 & $\sqrt{ }$ & 53 & 74 & $\sqrt{ }$ & 06 & 05 \\
\hline 9 & $\sqrt{ }$ & 45 & 69 & $\times[3]$ & 31 & -0.04 \\
\hline 10 & $\sqrt{ }$ & 62 & 84 & $\sqrt{ }$ & 14 & 15 \\
\hline
\end{tabular}

This case has taken as a result of several things wherever only a partial part of an image is offered and it is troublesome to recognize. In table-4, the performance that has shown in the result of the DoC is satisfactory and superior compare to Histogram.

The result of another sample case is shown below

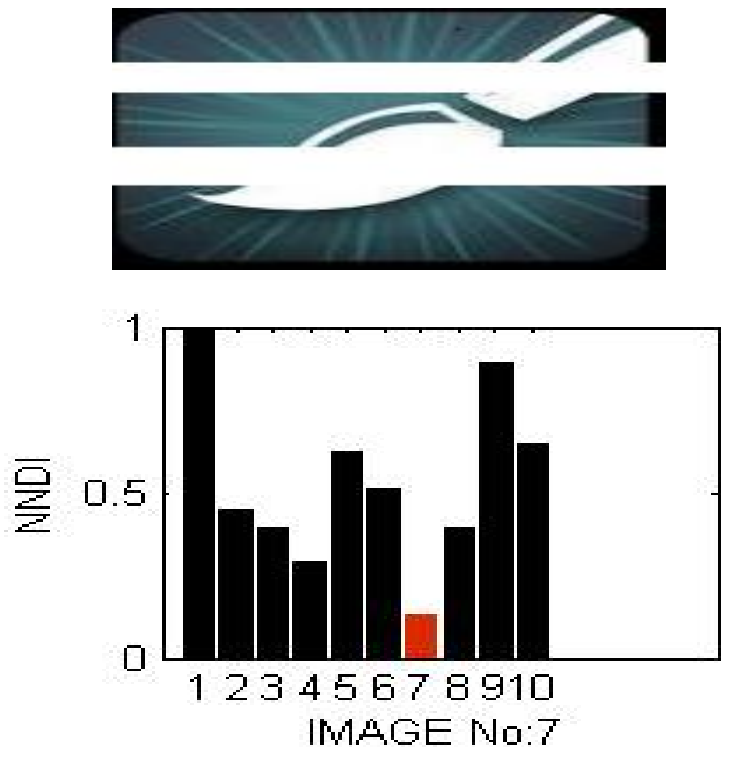

Fig 10. Recognition by DoC for image-vii

Fig 11. Recognition by Histogram method for image-vii

\section{E. Analysis of Fault-Tolerance}

Different positions of the faults and nature of faults are defined at random through uniform distribution for hidden layer weight connections (HC) and output connections (OC). Recognition can outline with these faulty connections for all images.

Table 5. Possible Faults In Weights

\begin{tabular}{|l|l|l|l|l|}
\cline { 3 - 5 } \multicolumn{2}{|c|}{} & Type 1 & Type 2 & Type 3 \\
\hline $\begin{array}{l}\text { ANN } \\
\text { LAYER }\end{array}$ & $\left|\mathrm{W}_{\max }\right|$ & $+\left|\mathrm{W}_{\max }\right|$ & $-\left|\mathrm{W}_{\max }\right|$ & Zeros \\
\hline HC & 1.0721 & +1.0721 & -1.0721 & 0 \\
\hline OC & 2.5085 & +2.5085 & -2.5085 & 0 \\
\hline
\end{tabular}

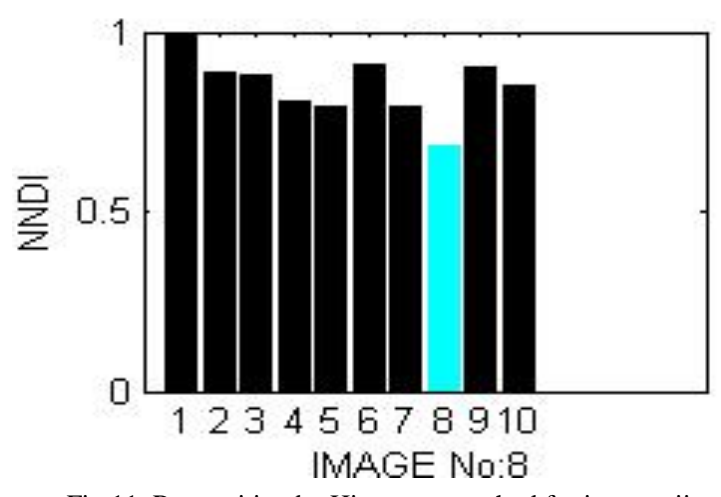


Table 6. Faults In Selected Position

\begin{tabular}{|l|llllllll|}
\hline Image & \multicolumn{3}{|l|}{ Original Weights } & \multicolumn{4}{ll}{ Fault Type } \\
\hline 1 & -0.161, & 0.481, & 0.678, & 0.303 & 2 & 1 & 3 & 1 \\
\hline 2 & 0.219, & 0.034, & 0.709, & 0.911 & 2 & 1 & 3 & 1 \\
\hline 3 & -0.246, & -0.940, & 0.922, & -.043 & 2 & 1 & 3 & 1 \\
\hline 4 & -0.765, & -0.231, & -0.537, & -.540 & 2 & 1 & 3 & 1 \\
\hline 5 & 0.144, & 0.146, & 0.567, & 0.492 & 2 & 1 & 3 & 1 \\
\hline 6 & 0.378, & -0.945, & 0.809, & 0.596 & 2 & 1 & 3 & 1 \\
\hline 7 & 0.632, & -0.744, & -0.537, & -0.278 & 2 & 1 & 3 & 1 \\
\hline 8 & 0.995, & -0.340, & -0.008, & 0.513 & 2 & 1 & 3 & 1 \\
\hline 9 & -0.454, & -0.218, & 0.537, & 0.874 & 2 & 1 & 3 & 1 \\
\hline 10 & 0.863, & 0.250, & 0.089, & 0.355 & 2 & 1 & 3 & 1 \\
\hline
\end{tabular}

In table-5, various sorts of potential fault values have been shown for each place of connections. In table-6, 4 different faults outlined at random in input-hidden layer connections. Amendment in every weight for every image has stated clearly and the performance is acknowledged with faulty weights, further more related condition has also specified for 10 faults and shown in table -7 .

Table 7. Performance With Faults

\begin{tabular}{|c|c|c|c|c|c|c|}
\hline \multirow{2}{*}{$\begin{array}{l}\text { Image } \\
\text { No. }\end{array}$} & \multicolumn{3}{|c|}{$\begin{array}{l}\text { Four Faults }[\text { Table-4] } \\
(\%)\end{array}$} & \multicolumn{3}{|c|}{ Ten Faults [Table-7] (\%) } \\
\hline & Rec. & RDMI & NNDI & Rec. & RDMI & NNDI \\
\hline 1 & $\sqrt{ }$ & 99 & 55 & $\sqrt{ }$ & 98 & 54 \\
\hline 2 & $\sqrt{ }$ & 92 & 57 & $\sqrt{ }$ & 83 & 43 \\
\hline 3 & $\sqrt{ }$ & 92 & 65 & $\sqrt{ }$ & 65 & 23 \\
\hline 4 & $\sqrt{ }$ & 91 & 66 & $\sqrt{ }$ & 90 & 52 \\
\hline 5 & $\sqrt{ }$ & 89 & 60 & $\sqrt{ }$ & 70 & 29 \\
\hline 6 & $\sqrt{ }$ & 72 & 41 & $\sqrt{ }$ & 72 & 45 \\
\hline 7 & $\sqrt{ }$ & 42 & 17 & $\sqrt{ }$ & 55 & 29 \\
\hline 8 & $\sqrt{ }$ & 60 & 35 & $\lambda[6]$ & & \\
\hline 9 & $\sqrt{ }$ & 90 & 51 & $\lambda[6]$ & & \\
\hline 10 & $\sqrt{ }$ & 85 & 60 & $\sqrt{ }$ & 78 & 50 \\
\hline
\end{tabular}

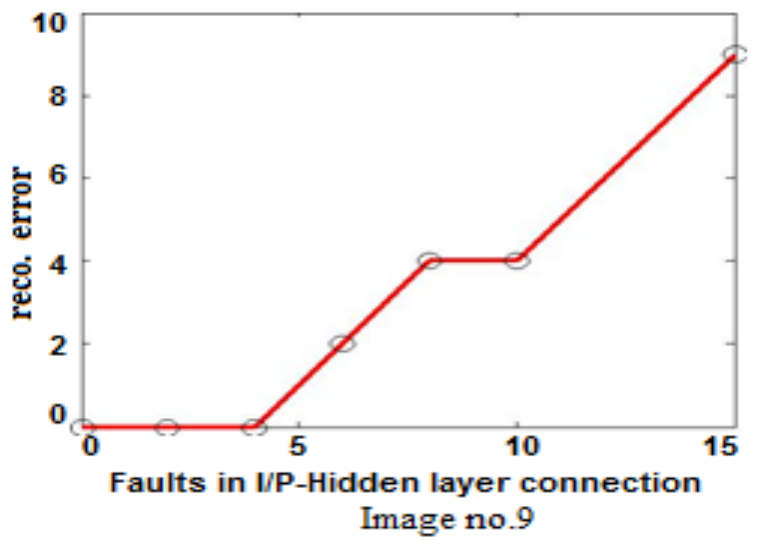

Fig- 12. DoC recognition with connection faults

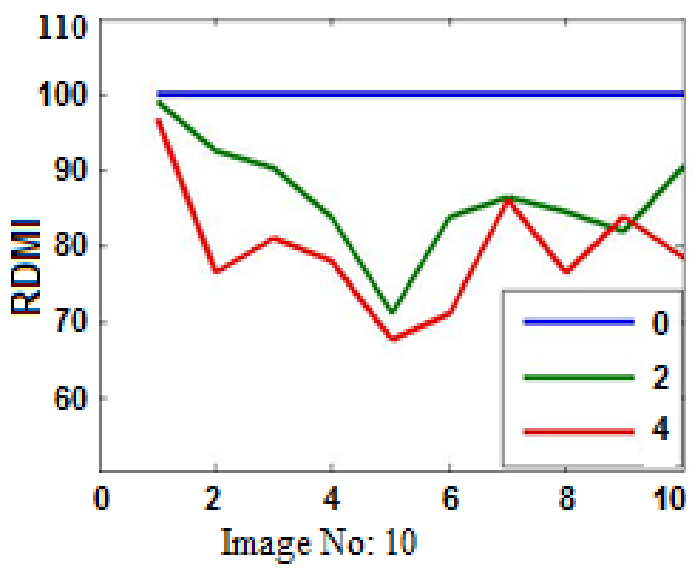

Fig 13. RDMI for successful recognition with connection faults

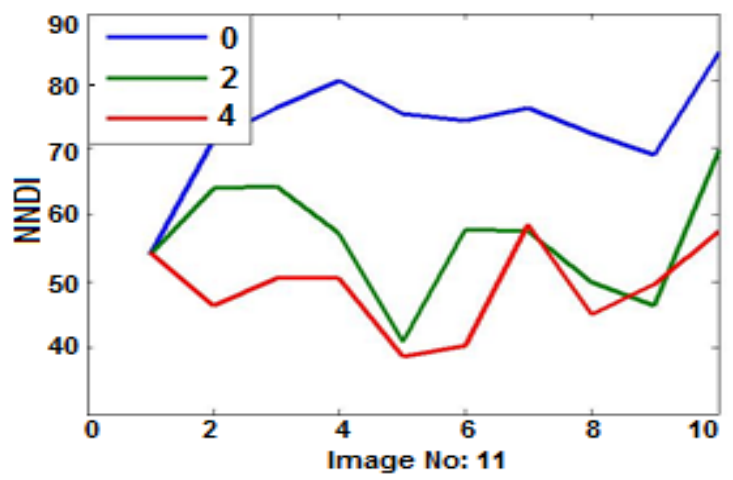

Fig 14. NNDI for successful recognition with connection faults

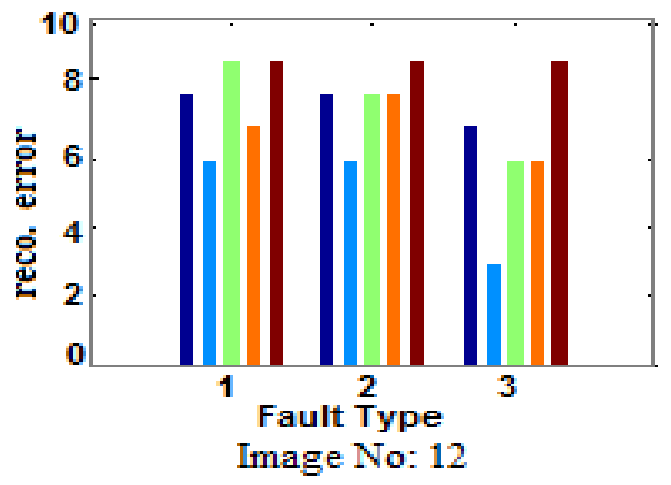

Fig 15. Performance with output layer connection faults

Nature of faults is all the time randomly, therefore in another experiment for various ranges of faults $[0,2,4,6$, 8,10 , and 15] category and place of the faults outlined subjectively. For every range of faults, the process has been repeated for five times independently to capture the more practical possibility. With 0,2 , and 4 faults, all the images are recognized successfully, whereas in other cases like 6,8,10, and 15, error in total recognition is 2, 4, 4 and 15, as shown in Fig-12. The faults 0,2 , and 4 achieve a mean value of RDMI and NNDI is also shown in Fig-13 and Fig-14.

To observe the impact of faults in output layer weights, three different types of faults are applied one by one for each connection (total 5) and recognition mechanism is applied to all the images as shown in table-5. Error in the 
recognition has shown in Fig-15 and it puts together the terribly clear concerning sensitivity of recognition w.r.t output connection. This sensitivity is exceptionally logical and zilch uncommon as a result the final output directly depends on these connection weights. Of course, it is not a considerable issue due to 2 reasons, first the probability of fault is very less $(0.1811)$ and second technical based particular action (like better material in connection) will be offered with these minute interconnections without disturbing the cost factor much.

\section{F. Custom Recognition}

Threshold depends on the appliance and tolerance limit of subjective demand that is outlined for recognition. The images which have a total error in DoC lesser than the threshold value are the feasible solutions among whichever, contain the min value of error taken as a final solution, otherwise it will be confirmed as new information. In the Fig-16, the neural network has 20 faults in hidden weights and applied to recognize the image 2 with threshold value equal to 4 and it is clear that the recognition is accurately defined, whereas for threshold value equal to 2 , no recognition is defined and as shown in Fig-17.

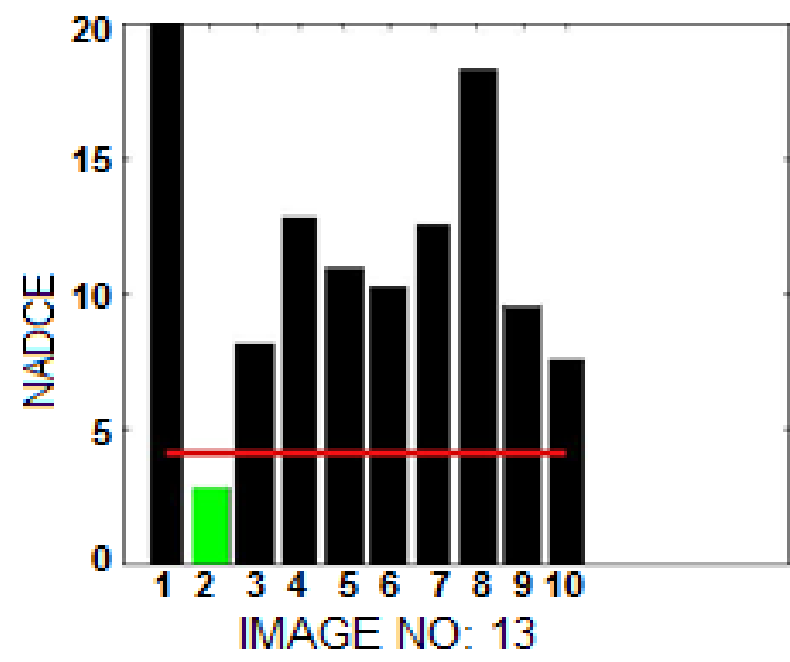

Fig 16. Custom Recognition for image 2 with $\mathrm{THR}=4$

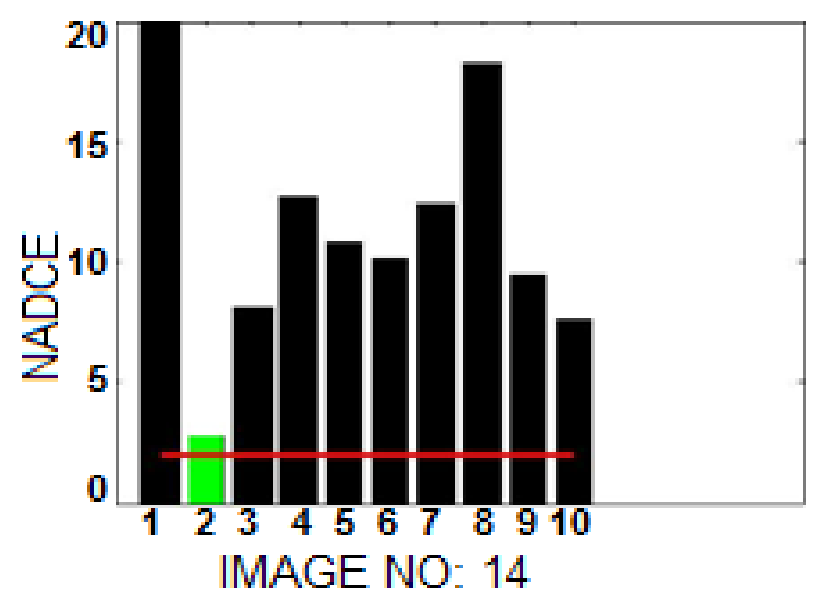

Fig 17. Custom Recognition for image 2 with $\mathrm{THR}=2$

\section{AdVANTAGES}

i. Here it is not required to store the image in its original form for recognition. Actually, only an array of DoC and trained weight connection matrix are stored for every image, therefore remarkable saving in memory requirement and less computations involved with significant processing speed.

ii. Stored information is well protected by default due to ANN approach, it is almost not possible to get the correlation and weights of an image from stored database, actually whole recognition process can be taken as "blind recognition".

iii. Robust against different kinds of noise applied in the test image due to nonlinear sigmoid function.

iv. Robust against numerous faults within the system.

v. Implementation is very easy and cost effective.

Properties (i) and (ii) are vital from the applications perspective, particularly for most web based applications or in data processing wherever storage, speed and security are the fundamental issues and should be attentive for rest of the options. Any abnormalities in input data will effortlessly be handle by property (iii). In hardware comprehension of proposed principle of recognition, role of fault-tolerance is an extremely vital issue to outline the standard solution. Property (iv) makes the solution sturdier against all intricate and probable conditions.

\section{CONCLUSION}

This is extremely efficient and economical approach for recognition based on the innovative conception that is the degree of correlation (DoC) and has been presented in this paper to achieve the extensive preciseness and robustness in the results. Depending on the forced or customary recognition for varied circumstances, the quality of the solution is sustained and secured for storing information in a database that's being generated by the proposed system. It presents an idea of commencement of blind authentication in numerous applications. The proposed solution has inclination towards development of an associated application for fixed images based on image retrieval in data mining. Typically, by the comparison of image recognition and color histogram, it is noticeably quantified that the proposed solution is superior. Fault-tolerance is another advantageous feature in proposed technique which analyzes the connections with weights available in ANN architecture at various levels and it has been shown robustness against faults in input-hidden layer connections.

\section{REFERENCES}

[1] "Pattern Classification", Richard, Peter.E.Hart David G. Stork, Wiley Publications, Nov' 9, 2000.

[2] Om Preeti Chaurasia, "An Approach to Fingerprint Image Pre-Processing",I.J. Image, Graphics and Signal Processing, Vol.4, No.6, July 2012, PP: 29-35 DOI:10.5815/ijigsp.2012.06.05. 
[3] Helena Galhardas, Antónia Lopes, Emanuel Santos, "Support for User Involvement in Data Cleaning ", LNCS, Springer, Data Warehousing and Knowledge Discovery,Vol. 6862,2011,pp:136-151.

[4] Kalaivany Natarajan, Jiuyong Li, Andy Koronios, "Data mining techniques for data cleaning", Springer, Engineering Asset Lifecycle Management, 2010, PP:796804.

[5] Tee Leong Kheng; Collin, C.; Ong Siong Lee, "E-Clean: A Data Cleaning Framework for Patient Data ", Informatics and Computer Intelligence (ICI), Page (s): 63 68.

[6] Richard Y. Wang, Veda C. Storey, and Christopher P.Firth, "A Framework for Analysis of Data Quality Research", IEEE Transactions on knowledge and Data engineering, Vol.7, NO, 4,1995, PP: 623-640.

[7] Huang, K., Lee, Y.,and Wang,R. "Quality Information and Knowledge",Prentice Hall, Upper Saddle River: N.J.1999

[8] Kahn, B.K, Strong, D.M, and Wang, R. Y."Information Quality Benchmarks: Product and Service Performance". Commun. ACM, (2002).

[9] Y. Won and P. Gader, "Morphological Shared -Weight Neural Network for Pattern and Target Detection", University of Missouri-Columbia, 1995.

[10] Shailendra Kumar Dewangan, "Real Time Recognition of Handwritten Devnagari Signatures without Segmentation Using Artificial Neural Network”, I.J.Image, Graphics,and Signal Processing, Vol.5, No.4, April 2013, PP.30- 37, DOI: 10.5815/ijigsp.2013.04.04.

[11] "Face Recognition System Using Back Propagation Artificial Neural Networks", N. Revathy, Guhan, International Journal of Advanced Engineering Technology, Vol. III, Issue I, Jan - Mar, 2012/321-324.

[12] Ali Javed, "Face Recognition Based on Principal Component Analysis",I.J.Image, Graphics, and Signal Processing, Vol.5, No.2, February 2013, PP.38-44, DOI: 10.5815/ijigsp.2013.02.06.

[13] S. Marina, M. Gore, G. Soda.. And C. Society "Artificial neural networks for document Analysis and recognition", IEEE transactions on pattern Analysis and machine intelligence, vol. 27, no.1, pp. 23-35, Jan. 2005.

[14] Jose Luis Hidalgo, Salvador Espana, Marıa Jose Castro, and Jose Alberto Perez, "Enhancement and Cleaning of Handwritten Data by Using Neural Networks", IbPRIA 2005, LNCS 3522, pp. 376-383, 2005.

[15] Y. Won and P. Gader, "Morphological Shared-Weight Neural Network for Pattern and Automatic Target Detection”, University of Missouri-Columbia, 1995.

[16] Ghada Kattmah,Gamil Abdel Azim, "Identification Based on Mutual Information and Neural Networks", I.J. Image Graphics, and Signal Processing, Vol.5, No.9, July 2013, PP.50- 57, DOI: 0.5815/ijigsp.2013.09.08.

[17] "Novelty Detection in image recognition using IRF Neural Networks properties", Philippe Smagghe, Jean Luc Buessler, Jean - Philippe Urban, ESANN 2013proceedings, European Symposium on Artificia Neural Networks, Computational Intelligence and Machine Learning. Bruges (Belgium), 24-26Apr 2013.

[18] Qian Lin; Peng CAI; Feng Zhang;, "Image recognition via discrete Hopfield neural network", IEEE, International conference on Advances in Energy Engineering(ICAEE),June2010,pp:339 - 342.

[19] Mutter, K.N.; Kaream, I. I. A.; Moussa, H. A, "Gray Image Recognition Using Hopfield Neural Network With MultiBitplane and Multi-Connect Architecture",IEEE International Conference on Computer Graphics, Imaging and Visualization, July 2006, PP: 236 -242.
[20] Lisheng $\mathrm{Xu}$, "Pulse image recognition using fuzzy neural network", Expert Systems with Applications: An International Journal,Vol.36,Issue 2,March,2009.

[21] Cheng, F.; Chen, F.N.; Ying, Y.B.; "Image Recognition of Unsound Wheat Using Artificial Neural Network", IEEE, Second WRI Global Congress on Intelligent Systems (GCIS), Dec 2010, PP: $172-175$.

[22] Li Hanguang;Zhao Xiaoyu; Zheng Guansheng;"“Chemical Image Recognition Based on BP Neural Networks", IEEE, International Conference on Information Science and Engineering (ICISE), Dec. 2009, pp: $1191-1195$.

[23] Aizenberg, I.; Alexander, S.; Jackson, J.; "Recognition of Blurred Images Using Multilayer Neural Network Based on Multivalued Neurons "IEEE International Symposium on Multiple-Valued Logic (ISMVL), May2011, pp: 282287.

[24] Hoshino, K.; Igarashi, H.;"An image recognition based on neural oscillator network "IEEE International Joint Conference on Neural Networks (IJCNN), July2010, PP: $1-6$.

[25] Huanglin Zeng and Yao Yi,'Image Recognition Using Adaptive Fuzzy Neural Network and Wavelet Transform “, Rough Sets, Fuzzy Sets, Data Mining, and Granular Computing, LNCS, Volume 2639/2003, 590, springer.

[26] Jian Wang; Jingfeng Yang; Shaofa Li;Qiufang, "Number Image Recognition Based on Neural Network Ensemble", IEEE international conference on Natural Computation, 2007. ICNC 2007. Aug.2007pp:237-240.

[27] Bock, S.; Newsome, S.; Wang, Q.; Zeng, W.; Lin, X.; Lu, J.; "image: An Image Based Information Retrieval Application for the iPhone",IEEE,Consumer Communications and Networking Conference (CCNC), Jan. 2010, PP:1-2.

\section{Authors' Profile}

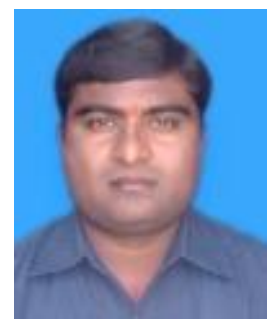

Mr. Raju Dara is a Research Scholar of Department of Computer Science and Engineering, Jawaharlal Nehru Technological University, Kakinada. He has 8 years of teaching experiences for Graduate and Post Graduate engineering courses. His current research interests are Data Warehousing, Image Processing. He published 5 research papers in international journals and 3 research papers in international conferences.

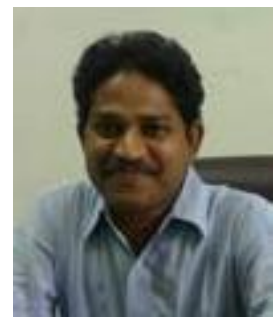

Dr. Ch. Satyanarayana is a Professor in Department of Computer science and Engineering at Jawaharlal Nehru Technological University Kakinada. He completed B. Tech and M.Tech in computer science and engineering from Andhra University, Vizag, A.P. He was awarded his Doctoral degree in 2008 from J.N.T. University, Hyderabad. He has 15 years of experience. His areas of interest are Image Processing, Databases, Pattern Recognition and Network Security. He published more than 21 research papers in international journals and more than 30 research papers in international conferences. Under his guidance 12 Research scholars are working on different areas like Image Processing, Speech Recognition, Pattern Recognition. He guided more than 
78 M.Tech Projects, 56 MCA Projects, and 36 B.Tech Projects.

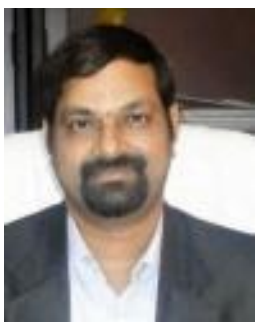

Dr.A.Govardhan is a Professor in Department of Computer science and Engineering at Jawaharlal Nehru Technological University, Hyderabad. He has 20 years of experience. His areas of interest are Data Warehousing \& Mining, Image Processing, and Databases. He did B.E. (CSE) from Osmania University College of
Engineering, Hyderabad in 1992, M.Tech from Jawaharlal Nehru University(JNU), New Delhi in 1994 and Ph.D from Jawaharlal Nehru Technological University, Hyderabad in 2003. He has 2 Monographs by Lambert Academic Publishing, Germany and Published in USA. He has guided $20 \mathrm{Ph} . \mathrm{D}$ theses, 125 M.Tech projects and he has published 152 research papers at International/National Journals/Conferences including IEEE, ACM, Springer and Elsevier.

How to cite this paper: Raju Dara, Ch.Satyanarayana, A. Govardhan,"A Novel Approach for Image Recognition to Enhance the Quality of Decision Making by Applying Degree of Correlation Using Artificial Neural Networks", IJIGSP, vol.6, no.11, pp.25-35, 2014.DOI: 10.5815/ijigsp.2014.11.04 